survival and proliferation of B cells and mTOR inhibition has been shown to be effective in immune $B$ cell suppression in transplant patients and in treatment of $B$ cell lymphomas. Interestingly, in a pSS mouse model mTOR targeting inhibited lymphocytic infiltration in the lacrimal gland. However, mTOR activation in B cells has not been studied in pSS patients.

Objectives: To study the mTOR pathway in B cells of pSS patients as a potential therapeutic target to inhibit $\mathrm{B}$ cell hyperactivity.

Methods: Expression of mTOR pathway genes (MTOR, RPTOR, RICTOR, DEPTOR, AKT1, IGF1R, IGF1, PTEN) were assessed on an OpenArray platform in purified peripheral blood B cells and monocytes from pSS patients $(n=12)$, non-Sjögren's sicca patients $(n=17)$ and healthy controls ( $H C, n=9)$. Correlations with clinical parameters including lymphocytic focus score, ESSDAI and serum IgG levels were assessed. Flow cytometry analysis for B cell subset distribution was performed to assess potential effects of $B$ cell subset distribution on gene expression differences. Culture experiments were performed to study inhibition of the mTORC1 pathway (phosphorylated S6, kinase activity downstream of mTORC1) in association with inhibition of B cell proliferation and IgG production by mTOR inhibition.

Results: RPTOR and IGF1R expression were significantly increased in B cells from $p S S$ patients $(p=0.019$ and $p=0.018$, respectively) and correlated with serum IgG levels $(r=0.429, p=0.020$, and $r=0.462, p=0.012)$. Differences in expression of mTOR pathway genes were not found in monocytes. To indicate the mTOR signature a cumulative mTORC1 score was calculated consisting of $Z$ scores (AKT1 + IGF1R + IGF1 + RPTOR + MTOR - PTEN - DEPTOR) which was significantly elevated in $p S S(p=0.027)$, correlating with serum IgG levels $(r=0.463$, $p=0.011$ ). Frequencies of memory and naïve $B$ cells did not differ between $p S S$ patients and $\mathrm{HC}$ in this cohort $(\mathrm{p}=0.415)$. Activation of $\mathrm{B}$ cells in culture resulted in phosphorylation of $\mathrm{S} 6$, which indicates increased mTORC1 activity, in accordance with $\mathrm{B}$ cell proliferation and IgG production in both $\mathrm{HC}$ and pSS patients. Inhibition of mTOR by rapamycin decreased $\mathrm{pS} 6(\mathrm{n}=4, \mathrm{n}=2 \mathrm{HC}, \mathrm{n}=2 \mathrm{pSS}$, MFI $1400 \pm 335$ vs $935 \pm 306, p=0.060)$, strongly reduced $B$ cell proliferation $(n=6, n=3$ HC, $n=3$ pSS, $80.8 \pm 9.9$ vs $19.1 \pm 15.8 \%, p<0.001)$, reduced IgG+ $B$ cells $(n=6, n=3 H C$, $n=3$ pSS $39.5 \pm 15.5$ vs $11.1 \pm 5.7 \%, p=0.001)$ and decreased IgG production $(n=4$, $\mathrm{n}=2 \mathrm{HC}, \mathrm{n}=2 \mathrm{pSS}, 160 \pm 180 \mathrm{pg} / \mathrm{mL}$ vs $25.3 \pm 13.0 \mathrm{pg} / \mathrm{mL} \mathrm{p}=0.060$ ).

Conclusions: Presence of an mTORC1 signature in B cells from pSS patients correlating with B cell hyperactivity indicates a role for $\mathrm{mTORC} 1$ in $\mathrm{B}$ cell activation in this disease. The fact that $B$ cell proliferation and $\lg G$ production is effectively inhibited by rapamycin suggests that mTOR inhibition represents a potential therapeutic strategy for pSS.

Disclosure of Interest: None declared

DOI: 10.1136/annrheumdis-2017-eular.1616

\section{THU0224 ASSOCIATION OF IRAK-M WITH NEUROPSYCHIATRIC SYMPTOMS IN SYSTEMIC LUPUS ERYTHEMATOSUS PATIENTS}

S. Sacre, R. Thwaites, K. Davies. Brighton and Sussex Medical School, University of Sussex, Brighton, United Kingdom

Background: Systemic lupus erythematosus (SLE) is a chronic systemic autoimmune disease, where a breakdown in immune tolerance leads to sustained inflammation and tissue damage. Patients exhibit multi-organ involvement with a diverse range of symptoms that include arthritis, nephritis, neuropsychiatric events and dermatological complaints. Interleukin-1 receptor-associated kinase $M$ (IRAK-M), a negative regulator of toll-like receptor (TLR) signalling has previously been associated with SLE in a murine study [1]. Deficiency of IRAK-M was observed to exacerbated disease in a SLE model in C57BL/6-Ipr/lpr mice [2].

Objectives: This study aimed to investigate the expression of IRAK-M in monocytes from SLE patients compared with healthy control donors and measure downstream cytokine production upon TLR stimulation.

Methods: The study was approved by the Brighton East Research Ethics Committee and the National Research Ethics Service Committee North West - Lancaster. Whole venous blood was collected from 39 SLE patients and 19 healthy donors. Peripheral blood mononuclear cells were purified from whole blood after which monocytes were isolated using $\mathrm{CD} 14^{+}$selection beads. Expression of IRAK-M was determined relative to the geometric mean of the housekeeping genes glyceraldehyde 3-phosphate dehydrogenase and hypoxanthine phosphoribosyltransferase 1 by quantitative polymerase chain reaction. Cultured monocytes were stimulated with TLR ligands. Cytokine production was measured by enzyme-linked immunosorbent assay.

Results: IRAK-M expression was increased in the SLE patient group, however many of the patients did not show elevated expression compared to healthy donors. When stratified by disease symptoms, a clear correlation was observed between low expression of IRAK-M and neuropsychiatric symptoms which was not evident when evaluating other SLE symptoms. Interestingly, TLR activation led to elevated cytokine production in SLE patient monocytes that correlated with the basal level of IRAK-M expression in individual donors. Further investigation revealed that SLE monocytes demonstrated a reduced upregulation of IRAK-M after TLR activation compared to healthy donors.

Conclusions: Increased expression of IRAK-M was not unexpected in the SLE group due to the systemic inflammatory nature of the disease. However, not all patients presented with increased levels of IRAK-M and upon activation were less able to upregulate IRAK-M compared to healthy donors, suggesting a reduced capacity to resolve inflammation. This was reflected in the elevated cytokine production observed from SLE monocytes. Thus, low expression of IRAK-M in SLE monocytes is linked to elevated inflammatory cytokine production and may be a biomarker for neuropsychiatric symptoms in SLE patients.

References:

[1] Kobayashi, K., et al. Cell, 2002. 110(2): p. 191-202.

[2] Lech, M., et al. Ann Rheum Dis, 2011. 70(12): p. 2207-17.

Acknowledgements: Study funded by Brighton and Sussex Medical School.

Disclosure of Interest: None declared

DOI: 10.1136/annrheumdis-2017-eular.6201

\section{THU0225 ROLE OF THE IL-12/IL-35 BALANCE IN SJÖGREN'S SYNDROME}

O. Fogel $^{1}$, E. Rivière ${ }^{2}$, R. Seror ${ }^{2}$, G. Nocturne $^{2}$, S. Boudaoud ${ }^{3}$, B. Ly ${ }^{3}$ J.-E. Gottenberg ${ }^{4}$, V. Le Guern ${ }^{1}$, J.-J. Dubost ${ }^{5}$, J. Nititham ${ }^{6}$, K. Taylor ${ }^{6}$,

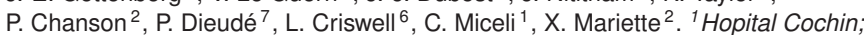
${ }^{2}$ Hopital Bicêtre; ${ }^{3}$ Inserm UMR1184, Paris; ${ }^{4}$ Hopital de Hautepierre, Strasbourg; ${ }^{5}$ Hopital Montpied, Clermont-Ferrand, France; ${ }^{6}$ UCSF, San Francisco, United States; ${ }^{7}$ Hopital Bichat, Paris, France

Background: An interferon (IFN) signature is involved in the pathogenesis of primary Sjögren's syndrome (pSS), but whether the signature is type 1 or 2 remains controversial. Mouse models and genetic studies suggested the involvement of Thelper 1 and type 2 IFN pathways. Likewise, polymorphisms of interleukin 12A gene (IL-12A), which encodes for IL-12p35, have been associated with pSS. IL-12p35 subunit is shared by 2 heterodimers, IL-12 and IL-35.

Objectives: To confirm the genetic association of $I L-12 A$ polymorphism and pSS and elucidate the involvement of the IL-12/IL-35 balance in pSS by functional studies.

Methods: The genetic study involved 673 patients with pSS from 2 French pSS cohorts and 585 healthy French controls. Functional studies were performed on sorted monocytes, stimulated or not. IL-12A mRNA and IL-12 and IL-35 protein levels were assessed by qRT-PCR and by ELISA and a multiplex kit for IL-35 and IL-12, respectively.

Results: We confirmed the association of the IL-12A rs485497 polymorphism and pSS and found an increased serum protein level of IL-12p70 in pSS patients carrying the risk allele $(p=0.016)$. Serum level of IL-12p70 was greater in patients than controls $(p=0.0001)$, especially patients with more active disease $(p=0.05)$; conversely IL-35 level was decreased in patients $(p=0.0001)$ especially in patients with a more active disease $(\mathrm{p}=0.05)$.

Conclusions: Our findings emphasize the involvement of the IL-12/IL-35 balance in the pathogenesis of pSS. Serum IL-35 level was associated with low disease activity, in contrast to serum IL-12p70 level, which was rather associated with a more active disease.

Acknowledgements: We thank Dr Odile Devergne (Université Paris Descartes, AP-HP, Hôpital Necker, Paris) for her expertise on the IL-35 ELISA kit to ensure the quality and reproducibility of this test.

The authors thank the following investigators of the ASSESS cohort prospective cohort of patients with Sjögren's syndrome (all in France) who recruited the patients and conducted follow-up: A. L. Fauchais (Limoges), S. Rist (Orleans), V. Le Guern (Paris), G. Hayem (Paris), J. Sibilia (Strasbourg), J. Morel (Montpellier), A. Saraux (Brest), A. Perdriger (Rennes), X. Puechal (Le Mans), E Hachulla (Lille) and V. Goeb (Rouen).

The authors thank Dr J. Benessiano, S Tubiana and all staff members of the Bichat Hospital Biological Resource Center (Paris) for their help in centralizing and managing biologic data collection from the French ASSESS,

Disclosure of Interest: None declared

DOI: 10.1136/annrheumdis-2017-eular.3586

\section{THU0226 DIFFERENTIAL SUSCEPTIBILITY OF TH17 AND T REGULATORY CELLS TO APOPTOSIS IN SYSTEMIC LUPUS ERYTHEMATOSUS PATIENTS - THE MODULATORY EFFECTS OF STATIN}

J. Sun, J. Frostegard, A. Liu. Institute of Environmental Medicine, Karolinska Institutet, Stockholm, Sweden

Background: Systemic lupus erythematosus (SLE) is a chronic autoimmune disorder. Patients with SLE have accelerated cardiovascular disease. Recent studies show there are more Th17 while less T regulatory (Treg) cells in the SLE patients. Th17/Treg imbalance may contribute to the pathogenesis of SLE.

Objectives: To investigate the underlying mechanisms of Th17/Treg imbalance, we test the proportion and susceptibility of Th17 and Treg to apoptosis, and the modulatory effects of statin in the SLE patients.

Methods: Totally 17 SLE patients and 20 gender- and age-matched control subjects were enrolled for this study. Peripheral blood mononuclear cells were isolated, either analyzed ex vivo, or cultured in the conditions to induce Th17 and/or Treg polarization. The proportion of Th17/Treg cells and frequency responding to apoptosis were analyzed by multiple color flow cytometry. Cytokines in cell culture supernatants and plasma were tested by ELISA. T cell polarization-related transcription factors were detected by quantitative real time PCR.

Results: The proportion of Th17 $\left(\mathrm{CD} 4^{+} \mathrm{IL} 17^{+}\right)$cells were higher in SLE patients, 
both in ex vivo and in the Th17-polarizing cultures. While the frequencies of Treg $\left(\mathrm{CD} 4^{+} \mathrm{CD} 25^{+} \mathrm{CD} 127^{\mathrm{dim} /-}\right)$ cells were lower in the corresponding populations. Higher levels of IL17 and IL6 were detected in plasma of SLE patients. Responding to CD95-induced apoptosis the frequency of $\mathrm{CD} 4^{+} \mathrm{IL} 17^{+}$cells from SLE patients was substantially lower, but that of $\mathrm{CD} 4^{+} \mathrm{CD} 25^{+} \mathrm{CD} 127^{\mathrm{dim} /-}$ cells from Treg-polarizing cultures was considerably higher. With treatment of atorvastatin, $\mathrm{CD} 4^{+} \mathrm{IL} 17^{+}$ cell population in cultures derived from SLE patients showed an increased susceptibility to CD95-induced apoptosis. However, the CD4 ${ }^{+} \mathrm{CD} 25^{+} \mathrm{CD} 127^{\mathrm{dim} /-}$ cell population had reduced response to apoptosis. Accordingly, the ratio of transcription factor RORC/FoxP3 decreased in T cell cultures of SLE patients. Conclusions: Th17 cells were more resistant than Treg cells to CD95-induced apoptosis in SLE as compared to control subjects. Statins counteracted the dysregulated susceptibility of SLE T cells to apoptosis. Our findings reveal a novel mechanism underlying the imbalance of Th17/Treg and show a potential interest to the treatment of the patients with SLE.

Disclosure of Interest: None declared

DOI: 10.1136/annrheumdis-2017-eular.6193

\section{THU0227 GENOME-WIDE ASSOCIATION META-ANALYSIS IDENTIFIES FIVE NEW LOCI FOR SYSTEMIC LUPUS ERYTHEMATOSUS}

A. Julià ${ }^{1}$, P. Carreira ${ }^{2}$, R. Blanco ${ }^{3}$, F.J. López-Longo ${ }^{4}$, J.J. Pérez-Venegas ${ }^{5}$, A. Olivé $^{6}$, J.L. Andreu ${ }^{7}$, M.A. Aguirre-Zamorano ${ }^{8}$, P. Vela ${ }^{9}$, J.M. Nolla ${ }^{10}$, J.L. Marenco de la Fuente ${ }^{11}$, A. Zea ${ }^{12}$, J.M. Pego ${ }^{13}$, M. Freire ${ }^{14}$, E. Díez ${ }^{15}$, M. López-Lasanta ${ }^{1}$, M. López-Corbeto ${ }^{1}$, N. Palau ${ }^{1}$, R. Tortosa ${ }^{1}$, E. Trallero ${ }^{1}$, A. Aterido ${ }^{1}$, D. Absher ${ }^{16}$, R.M. Myers ${ }^{17}$, A. Fernandez-Nebro ${ }^{18}$, S. Marsal ${ }^{1}$ ${ }^{1}$ Grup de Recerca de Reumatologia, Vall Hebron Research Institute, Barcelona;

${ }^{2}$ Rheumatology Department, Hospital Universitario 12 de Octubre, Madrid; ${ }^{3}$ Rheumatology Department, Hospital Universitario Marqués de Valdecilla, Santander: ${ }^{4}$ Rheumatology Department, Hospital Universitario Gregorio Marañón, Madrid; ${ }^{5}$ Rheumatology Department, Hospital del SAS de Jerez de la Frontera, Cádiz; ${ }^{6}$ Rheumatology Department, Hospital Universitari Germans Trias i Pujol, Badalona; ${ }^{7}$ Rheumatology Department, Hospital Universitario Puerta de Hierro, Madrid; ${ }^{8}$ Rheumatology Department, Hospital Universitario Reina Sofía, Córdoba: ${ }^{9}$ Rheumatology Department, Hospital General Universitario de Alicante, Alicante; ${ }^{10}$ Rheumatology Department, Hospital Universitari de Bellvitge, Barcelona; ${ }^{11}$ Rheumatology Department, Hospital de Valme, Sevilla; ${ }^{12}$ Rheumatology Department, Hospital Universitario Ramón y Cajal, Madrid: ${ }^{13}$ Rheumatology Department, Hospital do Meixoeiro, Vigo; ${ }^{14}$ Rheumatology Department, Hospital Universitario A Coruña, A Coruña; ${ }^{15}$ Rheumatology Department, Hospital Complejo Asistencial Universitario de León, León, Spain: ${ }^{16}$ Absher Lab: ${ }^{17}$ Myers Lab, HudsonAlpha Institute for Biotechnology, Huntsville, United States; ${ }^{18}$ Rheumatology Department, Hospital Regional Universitario de Málaga, Málaga, Spain

Background: Recent genome-wide association studies (GWAS) have identified more than 50 loci associated with systemic lupus erythematosus but they explain less than $30 \%$ of the heritability of the disease. Meta-analysis including new populations can contribute to identify additional genetic risk factors.

Objectives: The aim of the presents study was to identify additional genetic risk loci for SLE.

Methods: We performed a meta-analysis using data from a recent large scale GWAS from 4,036 cases and 6,959 controls from Caucasian European ancestry [1] and a newly genotyped cohort of 907 SLE patients and 1,524healthy controls from Spain. Genetic association was tested at the single-marker level using linear regression and at the pathway-level using Fisher's modified method.

Results: Combining the two cohorts we identified genome-wide significant association $(\mathrm{P}<5 \mathrm{E}-8)$ at five new loci: three SNPs at intragenic regions and two intergenic loci at chromosomes $7 q 11.23$ (Manhattan plot for chromosome 7 is shown in Figure; new SLE risk region SNPs highlighted in green) and 17q21.31.

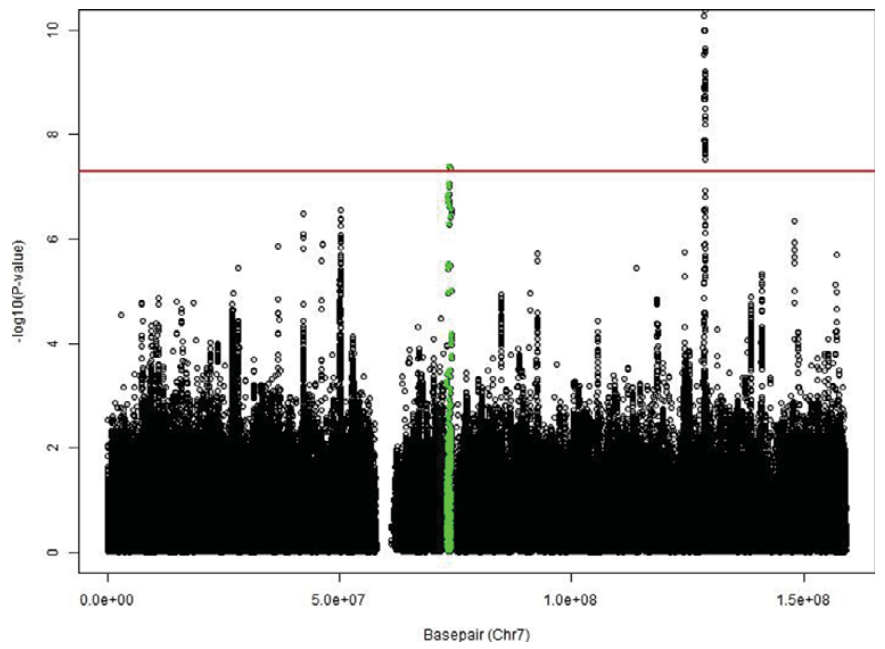

Several of the new associated genes are functionally associated with B cell regulation. After multiple test correction, $B$ Cell Receptor signaling, Biopeptides and Cell surface interactions at the vascular wall pathways were also significantly associated with SLE risk.

Conclusions: In conclusion, we have identified five new risk loci for SLE through a meta-analysis including a new GWAS.

\section{References:}

[1] Bentham J, Morris DL et al. Nature Genetics 2015

Acknowledgements: We would like to thank the clinical researchers and patients participating in the IMID Consortium for their collaboration. We would also like to thank the international SLE consortium for access to the data.

Disclosure of Interest: None declared

DOI: 10.1136/annrheumdis-2017-eular.2854

\section{THU0228 INTRA-RENAL ACTIVATION OF ADAPTIVE IMMUNE EFFECTORS IS ASSOCIATED WITH HIGHER DISEASE SEVERITY IN LUPUS NEPHRITIS}

C. Pamfil ${ }^{1}$, N. Demoulin ${ }^{2}$, M. Jadoul ${ }^{2}$, S. Aydin ${ }^{3}$, F.A. Houssiau ${ }^{1,4}$, B. Lauwerys $\mathrm{s}^{1,4}{ }^{1}$ Pôle de pathologies rhumatismales systémiques et inflammatoires, Institut de Recherche Expérimentale et Clinique, Université catholique de Louvain; ${ }^{2}$ Department of Nephrology; ${ }^{3}$ Department of Pathology; ${ }^{4}$ Department of Rheumatology, Cliniques universitaires Saint-Luc, Brussels, Belgium

Background: Chronic renal impairment remains a feared complication of lupus nephritis (LN). Yet, little is known about mechanisms and markers of disease severity in the lupus kidney.

Methods: We performed high-throughput transcriptomic studies (Illumina HumanHT12 v4 Expression Beadchips) on archived (- 80 degrees) kidney biopsies from 32 SLE patients and 8 controls (pre-transplant donors). Unsupervized clustering and differential gene expression studies were performed using GeneSpring software. Pathway analyses were carried on using DAVID and GSEA. Clinical and biological data were retrieved from the patients' medical records. Immunohistochemistry experiments (CD3, CD20, CD21) were performed on the same samples, and on an additional cohort of 37 SLE kidney biopsies. Syndecan-1 (SDC1) was used as a marker of renal tubular cell response to stress.

Results: Compared to controls, LN samples overexpressed transcripts involved in interferon signature, apoptosis, chemokines, antigen presentation, T and B cell activation

Unsupervized clustering studies isolated 14 SLE samples based on their gene expression features. These samples were characterized by a significantly lower estimated GFR at the time of biopsy (T0) (50.7 versus $\left.97.4 \mathrm{ml} / \mathrm{min} / 1.72 \mathrm{~m}^{2}\right)$, but also at follow-up ( 49.1 versus $85.8 \mathrm{ml} / \mathrm{min} / 1.72 \mathrm{~m}^{2}$ ) compared to the other $\mathrm{SLE}$ samples. Yet, apparent renal disease duration at T0, disease duration at last follow-up (median 91.5 versus 86 months), double-stranded DNA antibody titers at T0 and other relevant characteristics (histological scores, proteinuria, numbers of subsequent flares) were not different between both groups.

From a transcriptomic point of view, these 14 samples were characterized by the overexpression of transcripts and pathways involved in adaptive immune responses: antigen presentation, $\mathrm{T}$ cell differentiation and $\mathrm{B}$ cell activation. Immunohistochemistry studies confirmed a significant association between the presence of CD3 and CD20 positive cells in the interstitial space and lower estimated GFR at baseline in the same, but also in an independent set of samples. The presence of CD3 and CD20 positive cells was also associated with lower SDC1 expression on renal tubular cells. Low SDC1 expression on renal tubular cells was strongly associated with impaired kidney function at baseline. Conclusions: LN kidney biopsy samples from patients with lower estimated GFR are characterized by the overexpression of transcripts pointing to the activation of a local antigen-dependent immune response. Activation of "second wave" immune effectors in the LN kidney is a known feature of the disease, and impacts kidney function through alterations in the function of renal tubular cells.

Acknowledgements: The research leading to these results has received support from the Innovative Medicines Initiative Joint Undertaking under grant agreement $n$ 115565, resources of which are composed of financial contribution from the European Union's Seventh Framework Programme (FP7/2007-2013) and EFPIA companies' in kind contribution

Disclosure of Interest: None declared

DOI: 10.1136/annrheumdis-2017-eular.3427 